Supporting information

\title{
Toxicity alleviation of carbon dots from roast beef after forming protein coronas with human serum albumin
}

Kangjing Liu ${ }^{\text {abcd }}$, Yukun Song ${ }^{\text {abcd }}$ and Mingqian Tan ${ }^{\text {abcd* }}$

${ }^{a}$ School of Food Science and Technology, Dalian Polytechnic University, Dalian116034, Liaoning, People's Republic of China

${ }^{b}$ National Engineering Research Center of Seafood, Dalian 116034, Liaoning, People's Republic of China

${ }^{c}$ Engineering Research Center of Seafood of Ministry of Education of China, Dalian 116034, Liaoning, People's Republic of China

${ }^{d}$ Collaborative Innovation Center of Seafood Deep Processing, Dalian Polytechnic University, Dalian 116034, People's Republic of China

*Corresponding author (Tel\& Fax:+86-411-86318657, E-mail: mqtan@dlpu.edu.cn, ORCID: 0000000275350035). Address: Qinggongyuan 1, Ganjingzi District, Dalian116034, Liaoning, People's Republic of China 

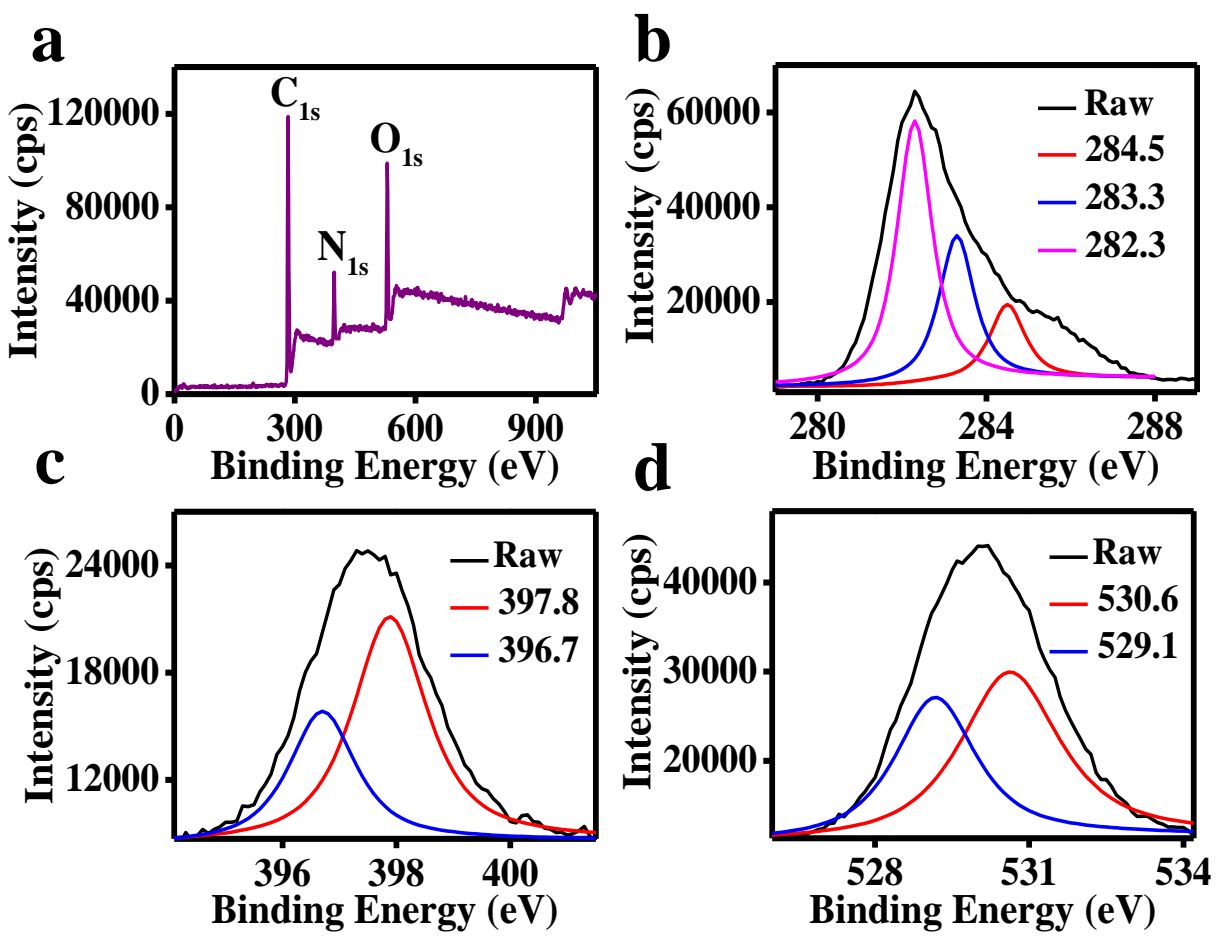

Figure S1. (a) XPS spectrum of the CDs. (b) High-resolution $\mathrm{C}_{1 \mathrm{~s}}$ spectra of the CDs. (c) Highresolution $\mathrm{N}_{1 \mathrm{~s}}$ spectra of the CDs. (d) High-resolution $\mathrm{O}_{1 \mathrm{~s}}$ spectra of the CDs. 

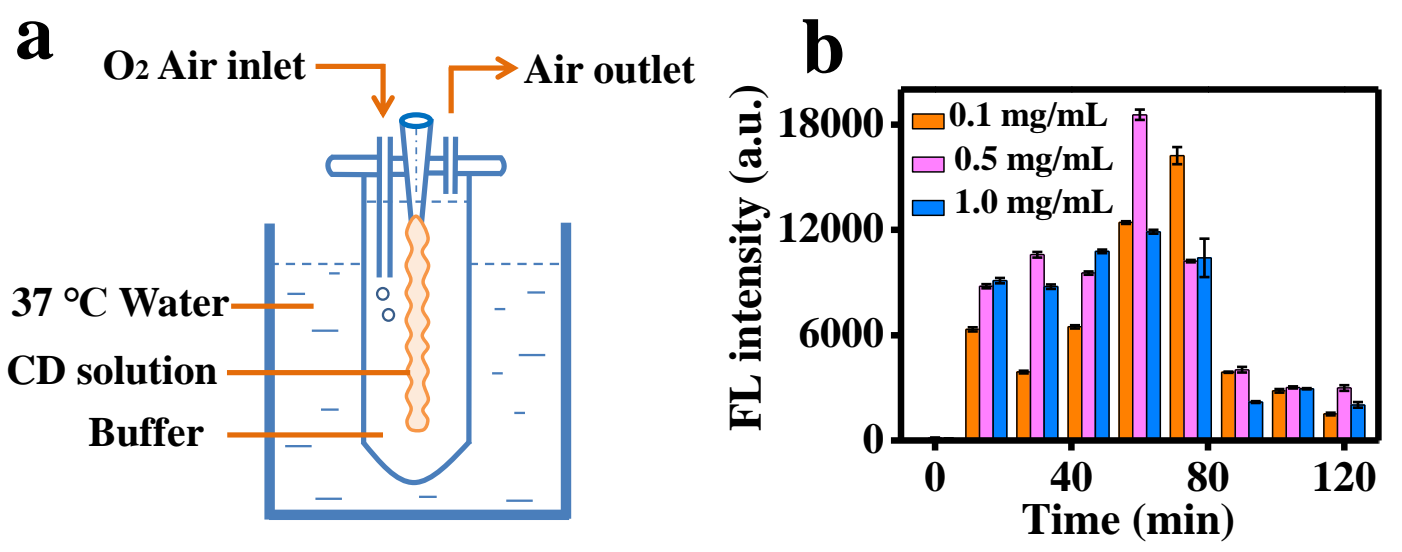

Figure S2. (a) Cartoon image shows the working mechanism of the everted rat gut sac model. (b) Histogram of parenteral CD fluorescence intensity when the concentration of CDs within intestine was $0.1,0.5,1.0 \mathrm{mg} / \mathrm{mL}$. 

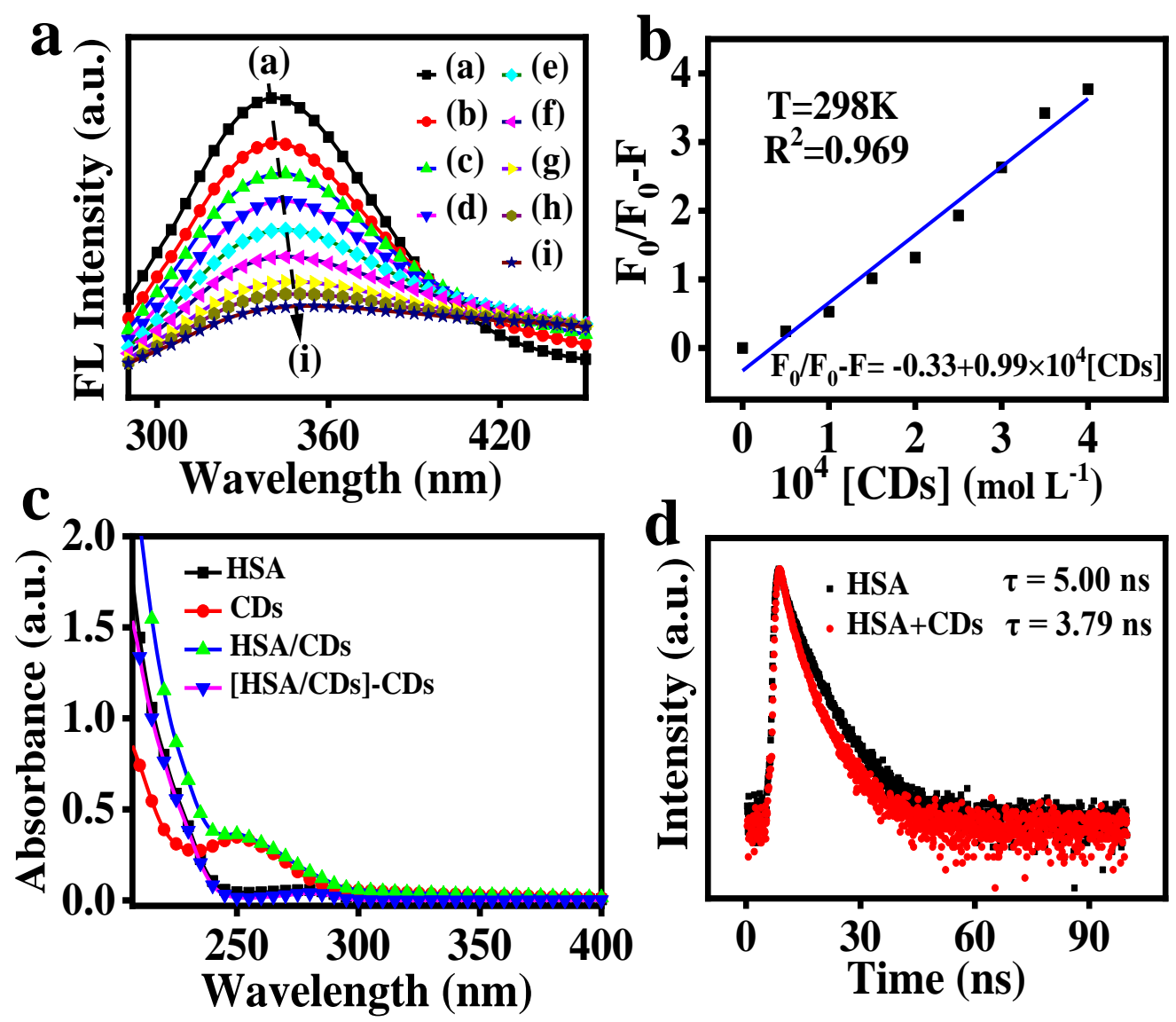

Figure S3. (a) Fluorescence internsity changes of HSA in the different concentrations of the CDs. $\mathrm{c}(\mathrm{HSA})=1 \times 10^{-5} \mathrm{~mol} / \mathrm{L}, \mathrm{c}(\mathrm{CDs}), \mathrm{a}-\mathrm{i}:$ 0, 0.5, 1.0, 1.5, 2.0, 2.5, 3.0, 3.5, 4.0 $\times 10^{-4} \mathrm{~mol} / \mathrm{L}, 298 \mathrm{~K}$. (b) Linear relationship between $\mathrm{F}_{0} / \mathrm{F}_{0}-\mathrm{F}$ value (HSA fluorescence quenching) and the $\mathrm{CD}$ concentration determined by Stern-Volmer equation at 298 K. (c) UV-Vis absorption spectra of the CDs, HSA, HSA-CDs and [HSA-CDs]-CDs. (d) Fluorescence decay curves of the CDs and HSA-CDs. 

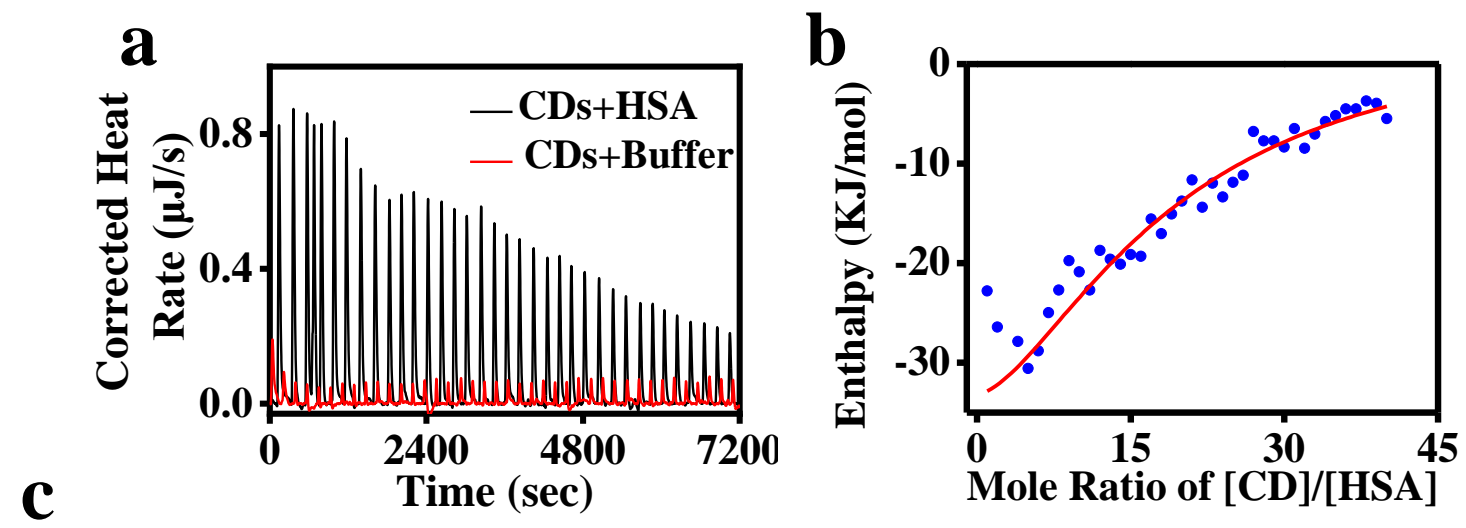

\begin{tabular}{|c|c|c|c|c|c|}
\hline$T(K)$ & $K_{d}\left(\times 10^{-4} / \mathrm{M}\right)$ & $n$ & $\Delta H(K J / m o l)$ & $\Delta G(K J / m o l)$ & $\Delta S(J / m o l / K)$ \\
\hline 298 & $5.93 \pm 0.47$ & $0.51 \pm 0.06$ & $-13.17 \pm 3.74$ & -21.53 & 28.04 \\
\hline
\end{tabular}

Figure S4. (a) Relationship of heat flow rate and injection time of the CDs to HSA of buffer (water) at 298 K. (b) Relationship between enthalpy and mole ratio of CDs at 298 K. (c) Thermodynamic parameters of the interaction between HSA and CDs. 


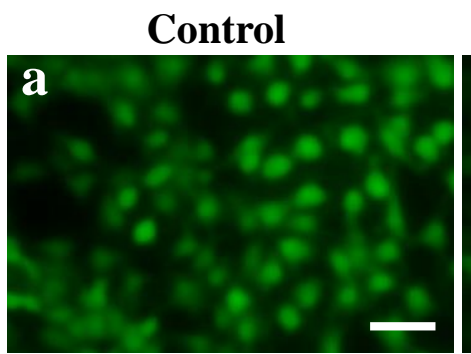

CDs

HSA-CDs

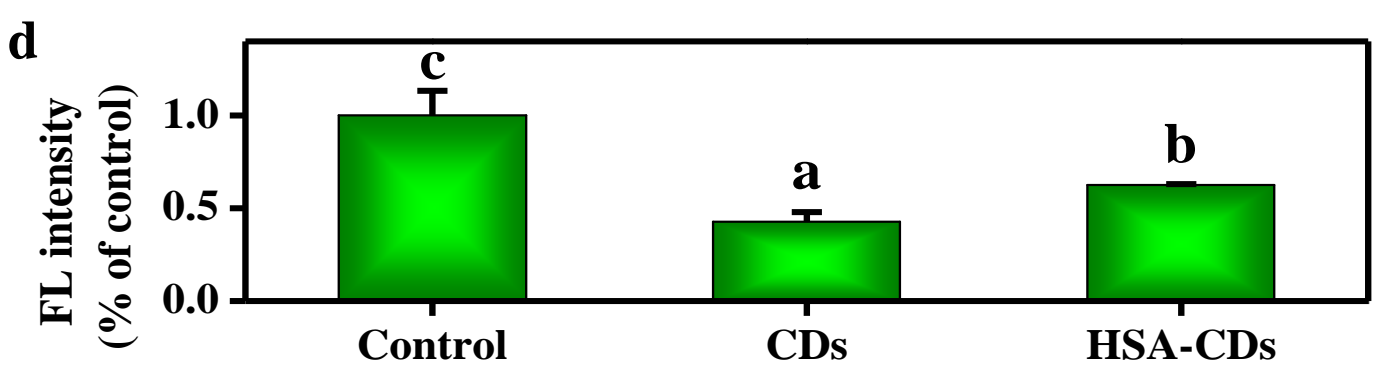

Figure S5. Fluorescent images of NRK cells stained with naphthalene-2, 3-dicarboxaldehyde after treatment of (a) DMEM without serum, $1 \mathrm{mg} / \mathrm{mL}$ of CDs in (b) the absence and (c) presence of $1 \times 10^{-5} \mathrm{~mol} / \mathrm{L}$ of HSA for $24 \mathrm{~h}$. (d) Relative fluorescence intensity of aphthalene-2, 3dicarboxaldehyde for (a) control, (b) CDs and (c) HSA-CDs. 